\title{
Heparin and a VKA - a winning combination
}

Following the serendipitous discovery of heparin by Jay McLean (MILESTONE 1) and the later isolation of the vitamin $\mathrm{K}$ antagonist (VKA) dicoumarol by Karl Link (MILESTONE 2) in the first half of the 20th century, considerable efforts were made to bring these newly identified anticoagulants from bench to bedside. Indeed, both heparin and the coumarin-derivative warfarin were in widespread clinical practice for the treatment of thrombosis by the 1950s, with the eminent example of US president Dwight D. Eisenhower being successfully treated with warfarin following a myocardial infarction in 1955.

By the late 1950s, a number of clinical studies had suggested that these anticoagulant drugs might be efficacious for the treatment of pulmonary embolism. Allen et al. reported in 1947 that only one death occurred in a cohort of 329 patients with pulmonary embolism who were treated with heparin and dicoumarol. In 1959, Bauer reported that only five deaths occurred from pulmonary embolism in a cohort of 627 patients with venous thrombosis who were treated with heparin. However, it was not until 1960 that the results of the first randomized clinical trial to evaluate these anticoagulants for the treatment of pulmonary embolism were published in The Lancet by Barritt and Jordan. This seminal study would have far-reaching implications for the field.

Based in the Departments of Medicine and Cardiology at the United Bristol Hospitals in Bristol, UK, Barritt and Jordan started patient enrolment for the trial in 1957. Patients with acute pulmonary embolism and no contraindication to anticoagulant therapy were randomly assigned - by card draw no less, demonstrating how far trial randomization has come in subsequent years
- to receive anticoagulation with heparin and the coumarin-derivative acenocoumarol, or no anticoagulant. The primary efficacy end point was death from pulmonary embolism, and the secondary end points were nonfatal recurrence and death from other causes.

In April 1958, an interim analysis was performed on the first 35 patients enrolled in the trial. Of

5) the 19 untreated patients, five had died from pulmonary embolism compared with no deaths in the 16 treated patients $(P=0.036)$. In addition, no recurrences occurred in the treatment group, compared with five nonfatal recurrences in the untreated group. As the improvements in composite outcomes at interim analysis were highly significant $(P=0.0005)$, Barritt and Jordan decided to admit all newly recruited patients to the treatment group thereafter; by July 1959, a total of 54 patients had been treated with anticoagulants. None of these patients died from pulmonary embolism, and only one patient had a nonfatal recurrence. Patients receiving anticoagulation had significantly better composite outcomes than untreated patients $(P=0.0000014)$.
This landmark study demonstrated for the first time that anticoagulation with heparin and a VKA markedly reduces the risk of death and recurrence of pulmonary embolism, paving the way for subsequent randomized clinical studies to evaluate these anticoagulants for the treatment and prophylaxis of venous thromboembolism (MILESTONE 4). As this therapeutic strategy remains the gold-standard treatment for venous thromboembolism to this day, Barritt and Jordan have undoubtedly had a long-term influence on the management of this condition, and have saved countless lives in the intervening $>50$ years.

Conor A. Bradley, Associate Editor, Nature Reviews Cross-Journal Team

ORIGINAL ARTICLES Allen, E. V. et al. The use of dicumarol as an anticoagulant: experience in 2,307 cases. Ann. Intern. Med. 27, 371-381 (1947)| Bauer, G. The introduction of heparin therapy in cases of early thrombosis. Circulation 19, 108-109 (1959) | Barritt, D. W. \& Jordan, S. C. Anticoagulant drugs in the treatment of pulmonary embolism: a controlled trial. Lancet 18, 1309-1312 (1960) FURTHER READING van Es, J. et al. Acute pulmonary embolism. Part 2: treatment. Nat. Rev. Cardiol. 7, 613-622 (2010) 